\title{
Wachstumsdifferenzierungsfaktor 15 ist ein hoch spezifischer Biomarker
}

Fragestellung: Ist die Bestimmung des Wachstumsdifferenzierungsfaktor 15 (GDF-15) der bestgeeignete Biomarker zum Nachweis einer mitochondrialen Erkrankung?

Hintergrund: Mitochondriale Erkrankungen sind mitunter sehr schwer zu diagnostizieren, da sie ein sehr buntes klinisches Bild aufweisen, wie zum Beispiel Muskelausdauerschwäche, Entwicklungsstörung, Kleinwuchs, Anfälle, Hörstörung, Störungen des Gleichgewichtes, Sehstörung, Diabetes, Leberfunktionsstörung und vieles mehr. Nicht immer sind Biomarker wie Laktat und Pyruvat im Serum weiterführend. Es wäre somit äußerst dienlich, vor der Durchführung einer Muskel-/Gewebebiopsie und genetischer Analyse einen leichter zu gewinnenden Biofaktor zu finden. 2011 wurde erstmals beschrieben, dass der Fibroblastenwachstumsfaktor 21 (FGF-21) ein erfolgversprechender Biomarker in Screening und Diagnose von muskelmanifestierenden mitochondrialen Erkrankungen darstellt. Somit wurde empfohlen, bei klinischer Verdachtsdiagnose den FGF-21 zu messen, dann eine genetische Aufarbeitung mit oder ohne histopathologischer und biochemischer Analyse einer Muskelbiopsie durchzuführen.

Der GDF-15 kommt in nahezu allen menschlichen Geweben vor. Er wurde bisher als Biomarker für Herz-, Nieren- und Krebserkrankungen vorgeschlagen. Bisher ist die Funktion von GDF-15 unklar. Es scheint aber ein Marker für erhöhte Sterblichkeit und Erkrankungen zu sein. Zudem kann es als Prognosemarker für die Entwicklung oder Besserung von Herz- und Nierenerkrankungen sowie

Yatsuga S, Fujita Y, Ishii A et al. Growth differentiation factor 15 as a useful biomarker for mitochondrial disorders. Ann Neurol 2015; $78: 814-23$ von manchen Krebserkrankungen sein. Die Autoren hatten im Vorfeld festgestellt, dass der GDF-15 bei Patienten mit MELAS (Mitochondrial encephalomyopathy, lactic acidosis, and stroke-like episodes) erhöht ist und wollten jetzt prüfen, ob diese GDF-15-Konzentrationserhöhung auch bei anderen Patienten mit mitochondrialen Erkrankungen eine sinnvolle Diagnosehilfe darstellt.

Patienten und Methodik: In diese Studie wurden 48 Patienten mit einer mitochondrialen Myopathie (mittleres Alter 34 Jahre) aufgenommen. Die meisten wiesen ein MELAS oder KearnsSayre-Syndrom auf. Dazu kamen 42 Patienten, die unter einer neurologischen Erkrankung wie zum Beispiel Duchenne Muskeldystrophie (sechs Patienten), Multiple Sklerose (neun Patienten), Aquaporin-positive Patienten (vier) litten. Diese wurden wiederum mit 146 Kontrollpatienten verglichen. Die Studie wurde am Universitätsklinikum Fukuoka durchgeführt.

Ergebnisse: Bei den Patienten mit einer mitochondrialen Erkrankung fanden sich sechsfach höhere GDF-15 Konzentrationen als bei gesunden Kontrollen $(2,711 \pm 2,459 \mathrm{pg} / \mathrm{ml}$ vs. 462,5 $\pm 141 \mathrm{pg} / \mathrm{ml}$ ). Dies war hoch signifikant. Die Trennschärfe zwischen Kontrollen und Patienten war in der Mehrzahl äußerst beeindruckend. Für Laktat, Pyruvat, Kreatinkinase und den Laktat/Pyruvat-Faktur galt dies nicht. Im Gegensatz zu GDF15 kam es bei der Bestimmung von FGF-21 zu einer Überlappung zwischen mitochondrialen Patienten und Kontrollen. Demgegenüber wiesen auch Patienten mit Duchennscher Muskeldystrophie, MS, optischer Neuritis und limbischer Enzephalitis deutlich niedrigere GDF-15-Spiegel auf. Somit erschien GDF-15 spezifischer und sensitiver. Bei der Analyse der Area under the curve war GDF-15 erneut deutlich besser in der Trennschärfe als FGF-21.

Schlussfolgerungen: Die Autoren folgerten aus ihren Ergebnissen, dass GDF-15 der bisher sensitivste Biomarker für eine mitochondriale Erkrankung ist.

\section{- Kommentar von Heinz Reichmann, Dresden}

\section{Sensitivster und spezifischster Test für mitochondriale Erkrankungen}

Diese Arbeit legt nahe, dass die Analyse von GDF-15 sensitiver und spezifischer ist, als die bisher proklamierte Bestimmung von FGF-21, das einen hohen Überlappungsbereich mit anderen Muskelerkrankungen aufweist. Eine naheliegende Empfehlung wäre somit, GDF-15 und FGF-21 mittels ELISA-Tests bei Patienten mit dem Verdacht auf mitochondriale Erkrankungen zu analysieren, um dann zu entscheiden, ob eine Muskelbiopsie durchgeführt werden sollte. Aus meiner Sicht handelt es sich in der Tat um den bisher sensitivsten und spezifischsten Test bezüglich mitochondrialer Erkrankung, wobei aber weder die Funktion noch die Gründe zur GDF-15-Erhöhung bekannt sind.

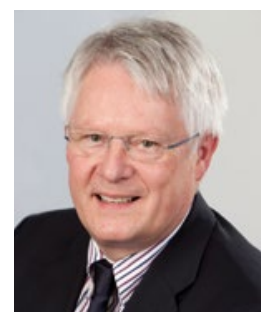

Prof. Dr. med. Heinz Reichmann, Dresden

Direktor der Klinik und Poliklinik für Neurologie, Klinikum Carl Gustav Carus der TU Dresden, Dekan der Medizinischen Fakultät. E-Mail: heinz.reichmann@ uniklinikum-dresden.de 